\title{
Design and technical validation of a wearable biofeedback system for robotic gait rehabilitation
}

\author{
Cristiana Pinheiro \\ Center for MicroElectroMechanical \\ Systems (CMEMS) \\ University of Minho \\ Portugal \\ ORCID: 0000-0002-7499-4162 \\ Luís M. Gonçalves \\ CMEMS \\ University of Minho \\ Portugal \\ E-mail: lgoncalves@dei.uminho.pt
}

\author{
João M. Lopes \\ CMEMS \\ University of Minho \\ Portugal \\ E-mail: a74982@alunos.uminho.pt \\ Cristina P. Santos \\ CMEMS \\ University of Minho \\ Portugal \\ E-mail: cristina@dei.uminho.pt
}

\author{
Joana Figueiredo \\ CMEMS \\ University of Minho \\ Portugal \\ E-mail: id6003@alunos.uminho.pt
}

\begin{abstract}
Gait disabilities affect the human quality of life. Current directions for time-effective robotic gait rehabilitation require the inclusion of biofeedback systems (BSs) as a complementary robotic tool for efficient motor relearning. This work aims to present the user-centered design and validation of a wearable BS to foster users' active participation and enable therapists' effective participation during robotic gait rehabilitation driven by active orthoses. The multimodal BS comprises a development board to manage the activation of the stimuli (vibrotactile through the vibrotactile waist and shank bands, sonorous via single earphone, and visual using RGB LED) according to data tracked by orthosis embedded sensors. The BS's versatility allows its functioning as a modular and stand-alone system or integrated into the orthotic system. The system's operability was validated with four healthy subjects walking on a treadmill with the orthotic system and BS at 1 $\mathrm{km} / \mathrm{h}$. The results showed an operable system with good usability during robotic gait rehabilitation. This wearable BS has the potential to boost symmetric gait recovery and to effectively augment the user's active participation during robotic gait therapy; thus, contributing to accelerating the user's motor recovery.
\end{abstract}

Keywords - human-robot interaction, robotic gait rehabilitation, user-centered design, wearable active orthoses, wearable biofeedback system

\section{INTRODUCTION}

Gait is a fundamental activity for locomotor independence and healthy daily life. Gait disabilities are mainly caused by central neurological disorders, affecting people worldwide. Around 5 million Europeans are wheelchair dependent. These victims present a high risk of falling, and they are unable to perform everyday tasks, compromising the quality of life. Paragraph supported by [1].

Current directions in gait rehabilitation aim for the integration of biofeedback systems (BSs) as complementary and alternative medicine therapy to conventional physical therapy or driven by active orthoses/exoskeletons/electrical stimulation [1]. In the context of gait rehabilitation, BS is an electromechanical device that includes sensors and stimulators to measure the user's motor activity (kinematic,

This work has been supported by the FEDER Funds through the Programa Operacional Regional do Norte and national funds from Fundação para a Ciência e Tecnologia with the project SmartOs under Grant NORTE01-0145-FEDER-030386, and through the COMPETE 2020-Programa Operacional Competitividade e Internacionalização (POCI)—with the Reference Project under Grant POCI-01-0145-FEDER-006941. kinetic and/or physiological parameters) and to, timely, provide this motor information to the user through visual, sonorous and/or haptic stimulation, respectively [2]. As posited by Wolf [3], the provided stimulus activates unused or underused neural central pathways to execute motor instructions, improving the patient's motor function and motor relearning.

The robotic assistive devices, such as active orthoses, are increasingly being integrated into everyday clinical practice. They offer an intensive, user-oriented and repetitive gait training, allowing efficient long-term gait rehabilitation. Autonomous and intelligent robotic systems like orthoses can guide the user's limbs according to a healthy gait pattern. In opposite to passive movements, patients should actively move towards human-orthosis interaction improvement. This active behaviour maximizes the involvement of the sensorimotor cortex, facilitating gait relearning. During robotic gait rehabilitation driven by active orthoses, BSs may prevent patients' motor function dependence on robotic devices, intuitively fostering the user's active participation and, consequently, accelerates motor recovery. Summary supported by [4]. Furthermore, during closed-loop gait training, BSs allow therapists to easily and accurately assess the patients' motor performance and, consequently, instruct the patients to adjust their movement accordingly, favouring gait recovery [5]. Otherwise, this information is too subtle to detect and too subjective to assess and manipulate accurately by a therapist [5]. Currently, in the literature, only two BSs were designed for robotic gait rehabilitation driven by active orthoses [5], [6]. None of these solutions is wearable and they do not involve an ankle-foot orthosis, limiting daily practice and ankle gait-related motion relearning, respectively. Resuming, there is a need to design wearable BSs for robotic gait rehabilitation driven by active orthoses [7].

This work aims to present the user-centered design and validation of a novel wearable BS to be used as a promising complementary tool of robotic gait rehabilitation driven by wearable ankle-foot active orthoses. Thus, it contributes to innovatively present a BS coupled to an ankle-foot orthotic system that enables ambulatory use and daily practice and fosters ankle gait-related motion relearning, encouraging user's active, and allowing therapist's effective participation. The system's requirements and the proposed solution are presented and technically described. Four biofeedback strategies, that explain how the stimuli are driven according to the tracked sensor information, are introduced. The system's 
validation included healthy subjects walking on a treadmill at $1 \mathrm{~km} / \mathrm{h}$ with the orthosis running the trajectory tracking control (operability study) and a system's usability questionnaire.

\section{METHODS}

\section{A. BS's requirements}

The technical and functional system requirements were defined by a multidisciplinary team of electronic and biomedical engineers and designers based on a list of endusers' requirements specified through a focus group. Thus, BS follows user-centered design principles to attain the user's necessities and maximize the user's satisfaction regarding the system's usability. The BS should comprise a user-oriented program to improve, progressively, the human-orthosis interaction. In this manner, the BS evolves low cognitive effort and allows gradual recovery which is supported by clinicians [8]. Moreover, the BS should comprise a therapistoriented program such that the therapists can, easily and accurately, follow users' performance. During robotic gait rehabilitation, this information cannot be assessed by the therapists through physical contact with the patients [5]. Therefore, the BS enables the therapists' effective participation in the therapy. They can encourage and motivate the patients, complementing the user-oriented program.

On the other hand, the requirements appoint to the BS to be/allow: (1) wearable (including wearable sensors and stimulators that fit most of the population) and stand-alone to permit ambulatory use and daily practice; (2) modular to enable its direct integration into different robotic devices; (3) time-effective response and to be robust to effectively foster the entire patient's motor recovery process; (4) easy to use and comfortable to achieve user's satisfaction; (5) multitasking so that the patient can perceive therapist's instructions (6) easily integrated into the orthotic system resulting in a compact system. The biofeedback strategies should be easily understandable and intuitive, allowing a short familiarization period and low cognitive effort.

\section{B. Proposed BS: system's overview}

According to system's requirements, the BS was designed to include four user-oriented biofeedback strategies to gradually improve the human-orthosis interaction: two biofeedback strategies to periodically teach the users when and how they should perform the paretic and non-paretic footfloor contact; two interaction-based biofeedback strategies to encourage the users to follow the orthosis direction and speed of movement. Moreover, the BS comprises one therapistoriented biofeedback strategy for each user-oriented biofeedback strategy, enabling therapist effective participation during therapy.

Fig. 1 shows an overview of the system. The BS's power supply is a battery (two rechargeable AA Ni-MH batteries) coupled to a step-up voltage, allowing stand-alone and compact features. The microcontroller (STM32F407VGT6 microcontroller) manages the data from the sensors embedded in the orthosis and runs the chosen biofeedback strategy, enabling the stimuli as needed. For user-oriented strategies, the stimulators are eccentric rotating mass (ERM) motors (Model 310-122, Precision Microdrives, United Kingdom), driven through specific drives (DRV2605L haptic driver), or earphones, which play a tone saved in the microcontroller's memory. For therapist-oriented strategies, the stimulator is a
Red-Green-Blue Light-Emitting Diode (RGB LED). The on/off state of the stimulus is transferred to and saved by the orthotic system to validate the correct functioning of the BS. The BS can be used as a module or integrated into the orthotic system. In the first case, the BS communicates with the orthotic system through an FT232R converter. In the second case, BS uses the orthotic system's microcontroller. The BS was developed to be wearable, robust, easy to use, comfortable, easily understandable, and to allow multitasking, following a user-centered design.

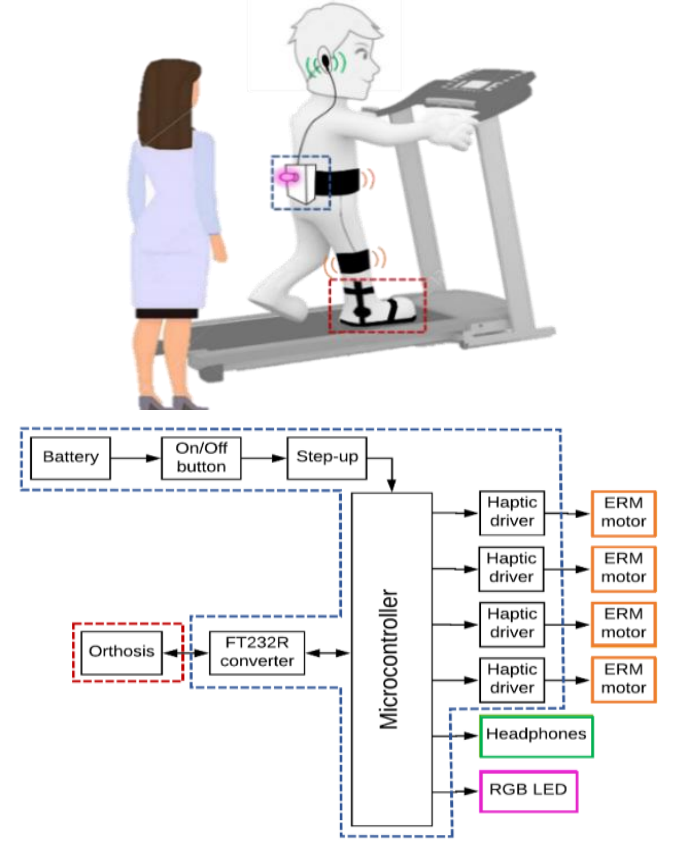

Fig. 1. Overview of the proposed system: the user timely receives vibrotactile or sonorous stimuli to improve the human-orthosis interaction (user-oriented biofeedback) and the therapist can effectively follow the user's performance through the RGB LED (therapist-oriented biofeedback).

\section{Orthotic system}

The presented orthotic system is a modular, wearable and innovative assist-as-need robotic system for robotic gait training of pathological users. This technological device allows repetitive gait training according to the user's needs and abnormal gait pattern correction. According to this, this robotic system is a powerful tool for pathological users to fast achieve functional motor recovery. It is composed by: (1) a wearable motion lab to monitor in real-time the patient's motor ability through ergonomic, stand-alone and wearable sensory systems; (2) an ankle-foot active orthosis, right-side module of the lower-limb H2-exoskeleton (Technaid S.L., Spain), to aid gait in the sagittal plane for gait speeds between 0.5 and $1.6 \mathrm{~km} / \mathrm{h}$; (3) power supply system (Fig. 2), a lithium iron phosphate battery with $8 \mathrm{~h}$ of autonomy and its hardware interface, to power up all the modules, allowing stand-alone and wearable features. Paragraph supported by [9].

The orthosis is composed by an electrical actuator (flat brushless DC motor EC60-100W, Maxon) coupled to a gearbox (CSD20-160-2A strain wave gear, Harmonic Drive), capable of providing an average torque of $35 \mathrm{Nm}$ and peak torque of $180 \mathrm{Nm}$, and embedded sensors. The embedded sensors are (1) potentiometer to measure the joint angle (resolution of $0.5^{\circ}$ ); (2) four strain gauges connected in a full Wheatstone bridge to measure the user-robot interaction torque (resolution of $1 \mathrm{Nm}$ ) (Fig. 2); (3) hall effect sensor to track the motor's angular speed, current and torque; (4) two 
force-sensitive resistors (FSRs), placed at heel and toe, to measure the ground reaction force [9].

The orthotic system (Fig. 2) has a bioinspired hierarchical control architecture with three levels (low-, mid- and highlevels) to generate assistive commands set by user-oriented assistive control strategies. The high-level control runs on a Raspberry Pi 3 board (Raspberry Pi Foundation, United Kingdom) which communicates through Universal Asynchronous Receiver-Transmitter (UART) with an STM32F4-Discovery board (STMicroelectronics, Switzerland). This board runs the mid- and low-level controllers and communicates with the ankle-foot active orthosis through Controller Area Network (CAN). In the scope of this work, the BS is validated with the ankle-foot active orthosis (Fig. 2) running the trajectory tracking assistive control strategy. During this assistive control strategy, the high-level control generates a healthy ankle joint trajectory for the sagittal plane adjusted according to the user's height and speed - user-oriented position trajectory. The mid-level controller sets the orthosis reference position trajectory as the user-oriented position trajectory. The low-level control is based on a close-loop proportional-integral-derivative (PID) controller tuned for gait speed ranged from 0.5 to $1.6 \mathrm{~km} / \mathrm{h}$. The output of the PID acts on the orthosis motor, generating a motor's torque, which in turn, acts on the user's joint to follow the reference trajectory. If the measured joint angle is not equal to the reference joint angle, caused by user-independent motion or reference trajectory variation, the controller corrects this angle, trying to impose a healthy gait pattern to the user. Every time that the user does not move according to the reference trajectory, an interaction torque between the user and robot results. Paragraph supported by [9].

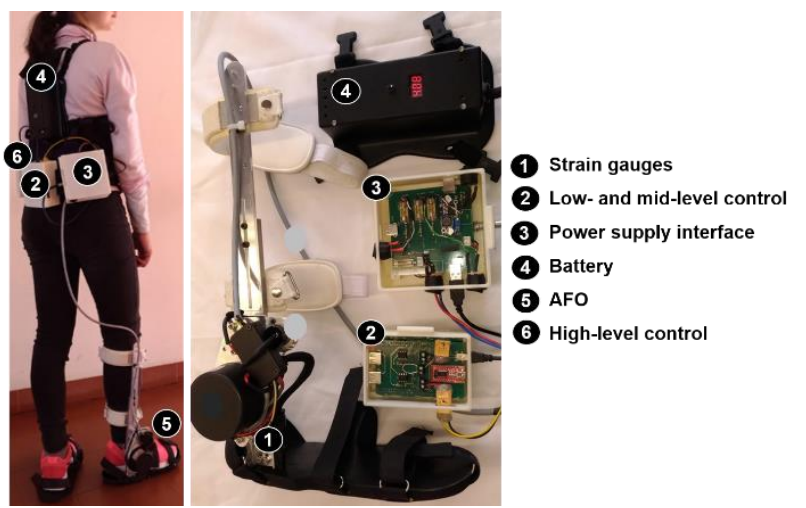

Fig. 2. Orthotic system with the embedded strain gauges, three levels of control (lower-, mid- and high- levels) and power supply.

\section{BS's components and interfaces}

The BS is a multimodal system, exploring three biofeedback modes: vibrotactile, sonorous and visual, provided through ERM motors, earphones and RGB LED, respectively. The vibrotactile and sonorous stimuli are fast perceived in comparison to the visual one, demanding less cognitive effort [10]. Therefore, the vibrotactile and sonorous stimuli were chosen for the user-oriented biofeedback strategies. The therapist-oriented biofeedback strategies are provided using visual stimulus.

Fig. 3 shows the BS's components and interfaces. The BS can be used as a module (Fig. 3B) or integrated into (Fig. 3A) the orthotic system, weighting $397 \mathrm{~g}$ and $387 \mathrm{~g}$, respectively. The first option (open-architecture) permits the use of BS with other robotic devices. The BS's versatility allows expanding its use in other applications and markets. In this case, the BS functions as stand-alone, entailing its power supply and development board, communicating with the orthotic system through FT232R USB to serial UART interface (FTDI, United Kingdom). The second option makes the orthotic system more compact, allowing easy to use and comfortable features. In this case, the BS uses the orthotic system's development board and its power supply.

As can be seen through Fig. 3, the electronics is centralized in a robust and ergonomic PCB $(119 \times 66 \mathrm{~mm})$, and it is protected by a solid and lightweight $3 \mathrm{D}$ printed box. The electronics should be allocated on the users' back through a waistband such that the system does not disturb the users' movements. Each BS's component is described below.

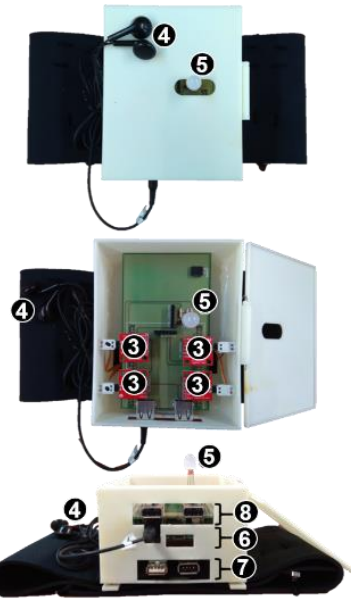

(A)

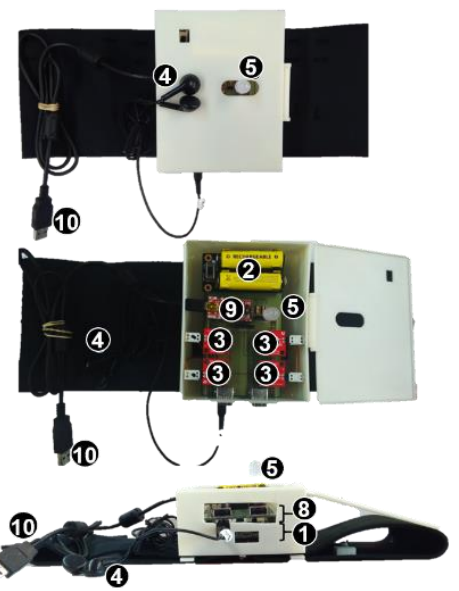

(B)

$\begin{array}{ll}\text { (1) Development board } & \text { 6 } \text { Development board (orthotic system) } \\ 2 \text { Power supply } & 7 \text { Orthotic system PCB } \\ 3 \text { Haptic driver } & 8 \text { Biofeedback PCB } \\ \text { 4 Earphones } & 9 \text { USB to serial UART interface } \\ \text { 6 RGB LED } & \text { 10 USB cable }\end{array}$

Fig. 3. BS's components and electronic interfaces. BS can be (A) integrated into or (B) a module of the orthotic system.

\section{1) Development board}

The STM32F4-Discovery board (STMicroelectronics, Switzerland) was chosen to be the development board of the BS, enabling the stimuli according to the selected biofeedback strategy. This board handles an STM32F407VGT6 microcontroller (32-bit ARM Cortex ${ }^{\circledR}$-M4 core) which can run at $168 \mathrm{MHz}$, has 1-Mbyte flash memory and 192-Kbyte RAM [11]. Also, it has embedded CS43L22 audio Digital-toAnalog Converter (DAC), class D speaker driver, InterIntegrated Circuit $\left(\mathrm{I}^{2} \mathrm{C}\right)$ and UART communication interfaces, and an adequate number of timers and $\mathrm{I} / \mathrm{O}$ pins [11]. Therefore, this board is prepared to produce a sound that can be listened through earphones. The $\mathrm{I}^{2} \mathrm{C}$ communication interface allows controlling the haptic drivers and CS43L22 audio codec. The UART communication interface allows the communication between the BS and the orthotic system when the BS is used as a module. All these features make the STM32F4-Discovery board a low-cost effective solution for this project. When the BS is used integrated into the orthotic system, the development board is also an STM32F4Discovery board.

\section{2) Power supply}

When used as stand-alone, the BS is supplied by two rechargeable AA Ni-MH batteries of $1.2 \mathrm{~V}$ and $2000 \mathrm{mAh}$ coupled to $5 \mathrm{~V}$ step-up voltage of $1 \mathrm{~A}$ maximum output current. This step-up has coupled support for the batteries, an on/off button and two LEDs to indicate the on and low battery 
states. This solution is compact and lightweight, contributing to the wearable feature of the BS. When the BS is integrated into the orthotic system, it is supplied by the wearable orthotic system's power supply. Therefore, the BS allows ambulatory use enabling daily practice.

\section{3) Vibrotactile stimulus-related components}

The coin shape ERM motors (Model 310-122, Precision Microdrives, United Kingdom) (Fig. 4) were chosen as vibrotactile stimulators since they are comfortable for onbody use and allow the modulation of the stimulus through the applied voltage [12]. Each ERM motor is regulated through a DRV2605L haptic driver (Texas Instruments, United States of America) due to the high current draw of the motors [13]. This driver accepts Pulse Width Modulation (PWM) signals as control, allows output voltage regulation and advanced driving techniques [13]. The vibrotactile motors are placed on the user's body through shank and waist textile bands. The shank has high haptic sensitivity [14], and the vibrotactile stimulus, in this body area, can stimulate the principal agonist muscles charged by ankle motion during gait [15], [16], facilitating the users' active participation. The waist is little susceptible to movement during gait [15], and the placement of the ERM motors in this area allows a compact BS since the remaining electronics are located on the user's back (Fig. 4). The vibrotactile bands are fundamentally constituted by neoprene, which gives elasticity to the bands. This elasticity allows the user's comfort and the bands to fit in different bodies and facilitates stimulus perception since the ERM motors can be very close to the skin. Small and large sizes were designed for each band to enable the use of the BS by most of the population.

In both waist and shank bands, the motors are equally spaced according to the reviewed haptic spatial resolution of shank and waist [14], respectively, to facilitate the stimulus spatial discrimination. Two ERM motors are placed on the shank: one at the front and one at the back over the tibialis anterior and soleus muscles, respectively (Fig. 4). Four ERM motors are placed on the waist: two at the front and two at the

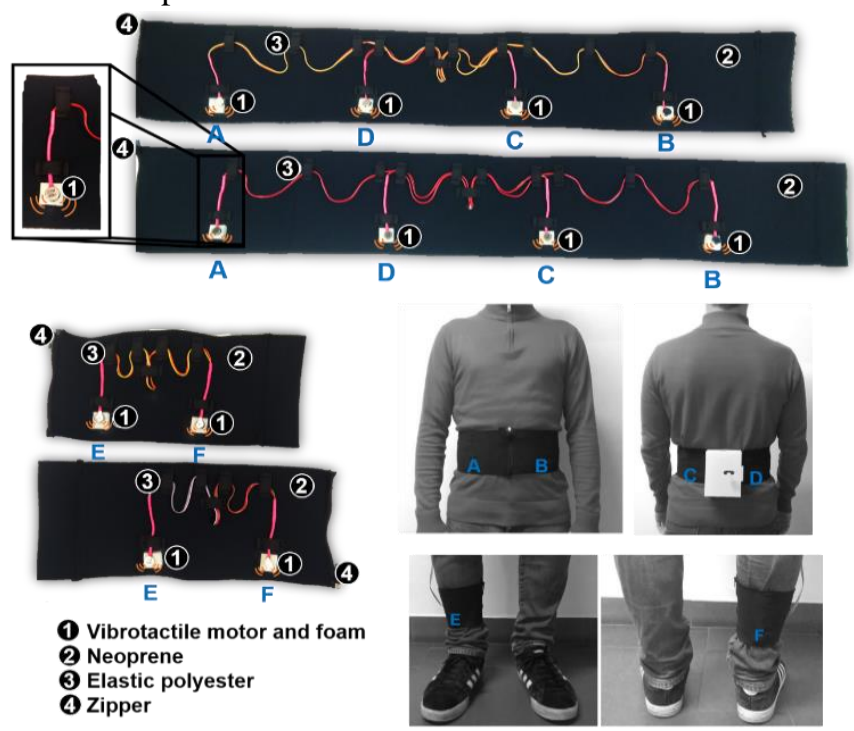

Fig. 4. Vibrotactile shank and waist bands (components and worn by a subject; $12 \mathrm{~cm}$ height and $3 \mathrm{~mm}$ thickness): small size (shank's lengths from $20 \mathrm{~cm}$ to $25 \mathrm{~cm}$; waist's lengths from $66 \mathrm{~cm}$ to $83 \mathrm{~cm}$ ) and large size (shank's lengths from $23 \mathrm{~cm}$ to $29 \mathrm{~cm}$; waist's lengths from $71 \mathrm{~cm}$ to $90 \mathrm{~cm}$ ). The foam prevents the propagation of the vibrotactile stimulus through the band, facilitating its spatial discrimination. The zig-zag configuration of the wires does not limit the elasticity of the bands. The zipper allows the bands to expand equally in a different direction so that the motors' location remains the same between users. back with symmetry between the left and right sides (Fig. 4). This configuration was chosen because the spatial discrimination is easier along the transverse axis of the body rather than the longitudinal axis [14]. Also, to place the ERM motors on the waist's right and left sides seems intuitive to discriminate the biofeedback for the paretic and non-paretic limbs, respectively. Moreover, two ERM motors can be enabled simultaneously to compensate for the lower haptic sensitivity of waist compared to shank [14].

\section{4) Sonorous stimulus-related components}

The earphones allow the users to listen to a sound without perturbing other people and they are wearable and small in opposite to speakers and screens with audio capability, respectively. Moreover, the user can use only one earphone, leaving the other ear completely available to listen to other sounds (e.g., advices and motivational statements from the therapists), permitting multitasking.

\section{5) Visual stimulus-related components}

The RGB LED is a small (diameter of $10 \mathrm{~mm}$ ), lightweight and wearable stimulator in contrast to screens (used in [5], [6]), which can be modulated through variation of light colour. The maximum luminous intensity is 600, 3000 and $750 \mathrm{mcd}$ for the colours red, green and blue, respectively. The diffuse feature of the LED and its orientation allows the therapists to perceive the visual stimulus from different directions (back, left and right sides of the users) (Fig. 4). It is placed on the user's back to not disturb them. The RGB LED was considered an easily understandable solution to help the therapists to follow the performance of the patients during rehabilitation.

\section{E. Biofeedback strategies}

The BS includes four user-oriented biofeedback strategies: (1) paretic foot-floor contact biofeedback to periodically teach the users when and how they should perform the paretic foot-floor contact along the gait cycle; (2) non-paretic foot-floor contact biofeedback to periodically teach the users when they should perform the non-paretic foot-floor contact along the gait cycle towards symmetric gait; (3) joint motion biofeedback to encourage the users to follow the orthosis direction of movement; (4) user participation biofeedback to encourage the users to follow the orthosis speed of movement.

During foot-floor contact biofeedback strategies, a stimulus is enabled according to the orthosis reference trajectory. The paretic foot-floor contact should start from heel-strike to flat-foot and stop from heel-off to toe-off of reference trajectory [15]. During non-paretic foot-floor contact biofeedback, a stimulus is activated so that patients can synchronize the non-paretic and paretic foot-floor contact towards symmetric gait, i.e., when the paretic limb performs heel-strike, the non-paretic limb should perform heel-off [15].

During interaction-based biofeedback strategies, a stimulus is enabled according to orthosis reference trajectory and human-orthosis interaction torque. During joint motion biofeedback, the interaction torque sign (positive, negative) should match reference trajectory monotony (decreasing, increasing) or baseline interaction torque should be achieved. During user participation biofeedback, the baseline interaction torque should be achieved. 


\section{F. Experimental validation}

Four healthy participants (age: $25.0 \pm 1.4$ years, height: $1.69 \pm 0.13 \mathrm{~m}$, body mass: $65 \pm 12 \mathrm{~kg}$ ) were recruited and signed a written informed consent to participate in the validation protocol of the BS. The participants were already familiarized with the orthosis trajectory tracking control strategy. They were instructed to walk on a treadmill at 1 $\mathrm{km} / \mathrm{h}$ three times during $1 \mathrm{~min}$, following the orthosis movement with the help of BS's cues (BS was used integrated into orthosis). Data concerning reference trajectory, interaction torque and on/off state of the biofeedback stimulus were acquired to conclude about the system's operability. Moreover, the comments of the participants and the person who supported them (with enough knowledge about the orthotic system and BS) during validation were registered, concerning their experience with the BS. In the end, the participants answered a questionnaire regarding the system's usability adapted from [17].

\section{RESUlTS AND DisCUSSION}

\section{A. System's operability}

Fig. 5 provides a representative example of one participant wearing the orthotic system and BS, and data acquired during the experimental validation. The results report the hardware functioning and the time-effective response of the stimulators according to sensors data.

During paretic foot-floor contact biofeedback strategy, the visual and vibrotactile stimuli (from waistband) were activated (Biofeed ON/OFF=1) from instant before the heelstrike event to the instant before the heel-off event. These events were defined according to the orthosis reference trajectory (Fig. 5A). Therefore, the BS is capable to inform the therapists and teach the users about when they should start and start the stop of paretic foot-floor contact, respectively. The information provided by the developed wearable BS is essential to the user accurately learn the healthy gait pattern, improving human-orthosis interaction. During non-paretic foot-floor contact biofeedback strategy, the visual and vibrotactile stimuli (from waistband) were on (Biofeed $\mathrm{ON} / \mathrm{OFF}=1$ ) from the instant before the heel-strike and heeloff to heel-strike and heel-off, respectively, of the reference trajectory (Fig. 5B). Thus, the BS can inform the therapists and teach the users about when they should perform the paretic and non-paretic limb's heel-strike, respectively, synchronizing the paretic and non-paretic foot-floor contact. This wearable BS showed to have the potential to boost the symmetric gait recovery that is still a challenge in unilateral robotic gait assistance [18].

During joint motion biofeedback strategy, the visual and sonorous stimuli were on (Biofeed $\mathrm{ON} / \mathrm{OFF}=1$ ) when the interaction torque is not the baseline and its sign does not match with the reference trajectory monotony (e.g., reference trajectory decreases - orthosis plantar flexion - and interaction torque is negative - user's dorsiflexion) (Fig. 5C). In this sense, the BS informs the therapists about users' performance while simultaneously encourages the users to follow the orthosis direction of movement (e.g., the user should perform plantar flexion during orthosis plantar flexion). This strategy is the mid-step to achieve maximum synchronism between orthosis (healthy gait pattern) and paretic limb. During user participation biofeedback strategy, the visual and vibrotactile stimuli (from shank band) were on (Biofeed ON/OFF=1) when the interaction torque is not the baseline and its sign matches with the reference trajectory monotony (e.g., reference trajectory increases - orthosis dorsiflexion - and interaction torque is negative - user's dorsiflexion) (Fig. 5D). Hence, the BS informs the therapists about users' performance and encourages the users to follow the orthosis speed of movement (e.g., users should adjust their dorsiflexion speed with the orthosis dorsiflexion speed). This strategy is useful to augment the user's active participation properly during gait therapy; thus, contributing to accelerating the user's motor recovery [1].

In summary, these results showed that the developed wearable BS is functionally operative (operative hardware and time-effective response) according to the pre-defined
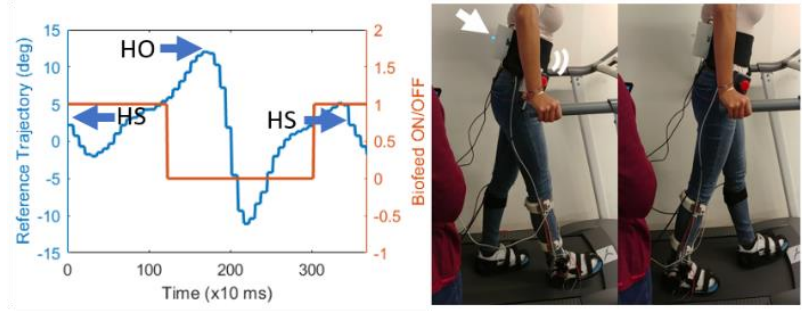

(A)

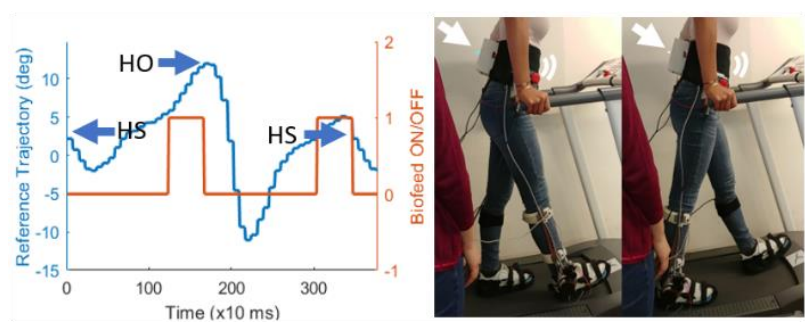

(B)
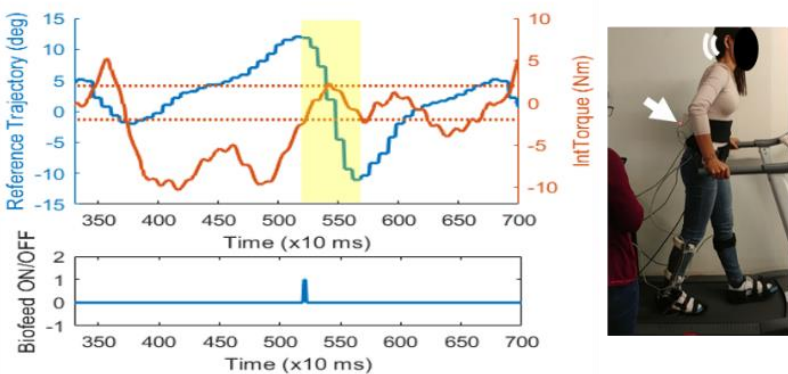

(C)

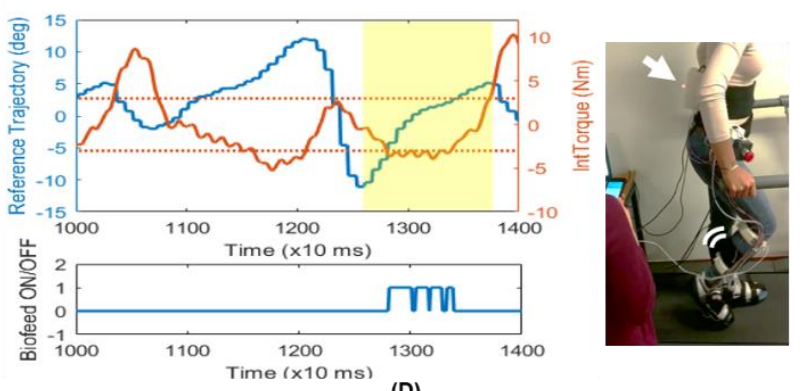

(D)

Fig. 5. Pictures of one participant and data acquired (reference trajectory in $\mathrm{deg}$, interaction torque in $\mathrm{Nm}$, on/off state of biofeedback stimuli) during the validation protocol. (A) Paretic and (B) non-paretic foot-floor contact strategies. Interaction-based strategies (baseline interaction torque dashed, actuation area marked as yellow): (C) joint motion and (D) user participation. HS and HO refer orthosis heel-strike and heel-off, respectively. 
operational requirements, and it is safely and reliably integrated into a robotic assistive device. In contrast with [5], [6], this BS is wearable and stand-alone, allowing ambulatory use and daily practice. Also, it presents an open-architecture permitting to expand its application by other orthotic systems.

\section{B. System's usability}

The participants commented that the vibrotactile waist and shank bands are comfortable and the stimuli are easily perceived. Also, they reported that is very intuitive to distinguish the biofeedback of each limb through the vibrotactile waistband. Relatively to the earphones, the participants mentioned that they do not disturb and allow multitasking. The person who supported the participants during the validation protocol referred that the RGB LED provides an easily understandable stimulus and it is very helpful to follow the users' performance, particularly during interaction-based biofeedback strategies, since it is difficult to perceive through visual inspection of users' limbs. Lastly, the participants commented that they feel more confident and motivated to use orthosis and more sensitive to the orthosis movement. According to the questionnaire's answers (Fig. 6), most of the participants reported that: if they need, they would like to use the BS frequently; the system's functioning is easily understandable; the system was easy to use; after understanding how the system works, they would like to have the support of a technical person along with the gait trial; the system is not uncomfortable to use. In summary, the system, that has followed a user-centered design (which is not shown in [5], [6]), showed to be useful and achieved users' satisfaction, proving its high usability.

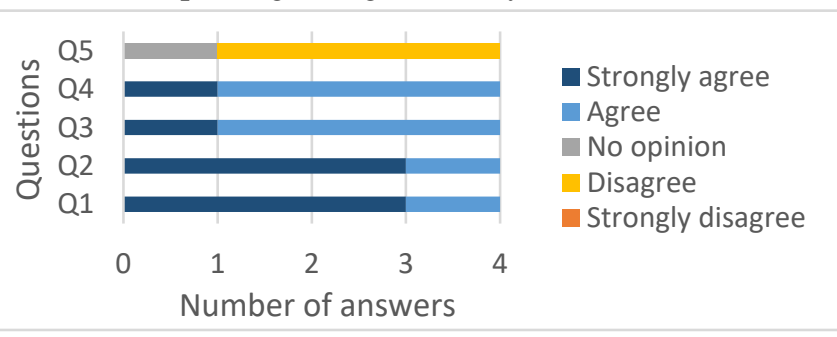

Fig. 6. Questionnaire's (Q1: if I need, I would like to use this system frequently; Q2: system's functioning is easily understandable; Q3: system was easy to use; Q4: after understanding how the system works, I would need the support of a technical person along with the gait trial; Q5: system is uncomfortable to use) answers (strongly agree/disagree, agree/disagree, no opinion) from the four participants about the system's usability.

\section{CONCLUSIONS}

This work presents a user-centered design of a wearable BS to be used during robotic gait rehabilitation driven by orthoses to foster patients' active participation and enable therapists' effective participation. The BS is multimodal, providing vibrotactile, sonorous and visual stimuli through ERM motors placed on elastic waist and shank bands, earphones and RGB LED, respectively. The STM32F4Discovery board manages the activation of these stimuli in real-time according to orthosis reference trajectory and human-robot interaction torque. The versatility of the BS allows its use integrated into or as a module of the orthotic system. The results from the validation protocol with four healthy subjects demonstrated an operable system with high usability. Future work comprises to study the system's effects in gait-related parameters, to convert the $\mathrm{BS}$ in a wireless device, clinical validation with gait injured patients and to expand the system's application by other orthotic and electrical stimulation systems.

\section{REFERENCES}

[1] T. Mikolajczyk et al., "Advanced technology for gait rehabilitation: An overview," Adv. Mech. Eng., vol. 10, no. 7, pp. 1-19, Jul. 2018.

[2] L. M. A. van Gelder, A. Barnes, J. S. Wheat, and B. W. Heller, "The use of biofeedback for gait retraining: A mapping review," Clin. Biomech., vol. 59, pp. 159-166, Nov. 2018.

[3] H. Huang, S. L. Wolf, and J. He, "Recent developments in biofeedback for neuromotor rehabilitation.," J. Neuroeng. Rehabil., vol. 3, p. 11, 2006.

[4] V. Klamroth-Marganska, "Stroke Rehabilitation: Therapy Robots and Assistive Devices," in Advances in Experimental Medicine and Biology, vol. 1065, 2018, pp. 579-587.

[5] O. Stoller, M. Waser, L. Stammler, and C. Schuster, "Evaluation of robot-assisted gait training using integrated biofeedback in neurologic disorders," Gait Posture, vol. 35, no. 4, pp. 595-600, Apr. 2012.

[6] F. Tamburella et al., "Influences of the biofeedback content on robotic post-stroke gait rehabilitation: electromyographic vs joint torque biofeedback," J. Neuroeng. Rehabil., vol. 16, no. 1, p. 95, Dec. 2019.

[7] C. Pinheiro, C. P. Santos, E. Garcia, and J. Figueiredo, "Biofeedback system to improve the human-orthosis interaction," University of Minho, 2019.

[8] J. Melin, "Patient Participation in Physical Medicine and Rehabilitation: A Concept Analysis," Int. Phys. Med. Rehabil. J., vol. 3, no. 2, pp. 36-42, Jan. 2018.

[9] J. Figueiredo, C. P. Santos, and J. C. Moreno, "Smart wearable orthosis to assist impaired human walking," University of Minho, 2019.

[10] A. H. S. Chan and A. W. Y. Ng, "Finger response times to visual, auditory and tactile modality stimuli," Lect. Notes Eng. Comput. Sci., vol. 2196, pp. 1449-1454, 2012.

[11] STMicroelectronics, "Discovery kit with STM32F407VG MCU," UM1472 User Man., 2017.

[12] P. Microdrives, "10mm Vibration Motor - 3mm Type," 310-122 Prod. Data Sheet

[13] Texas Instruments, "DRV2605L 2 to 5.2 V Haptic Driver for LRA and ERM with Effect Library and Smart-Loop Architecture," DRV2605L, 2014.

[14] K. Myles and M. S. Binseel, "The Tactile Modality: A Review of Tactile Sensitivity and Human Tactile Interfaces (Report No. ARL-TR4115)," Army Res. Lab., 2007.

[15] J. Perry, Gait Analysis: Normal and Pathological Function. SLACK Incorporated, 1992.

[16] A. Katusić and V. Mejaski-Bosnjak, "Effects of vibrotactile stimulation on the control of muscle tone and movement facilitation in children with cerebral injury.," Coll. Antropol., vol. 35 Suppl 1, pp. 57-63, 2011.

[17] B. J., "SUS: A quick and dirty usability scale," Usability Eval. Ind., vol. 189, pp. 4-7, 1996.

[18] P. Malcolm, S. Galle, P. Van den Berghe, and D. De Clercq, "Exoskeleton assistance symmetry matters: unilateral assistance reduces metabolic cost, but relatively less than bilateral assistance," $J$. Neuroeng. Rehabil., vol. 15, p. 74, Dec. 2018. 